\title{
Quantum Ratchets for Quantum Communication with Optical Superlattices
}

\author{
Oriol Romero-Isart \\ Departament de Física, Grup de Física Teòrica, \\ Universitat Autònoma de Barcelona, E-08193 Bellaterra, Spain. \\ Juan José García-Ripoll \\ Facultad de CC. Físicas, Universidad Complutense de Madrid, \\ Ciudad Universitaria s/n, Madrid, E-28040, Spain.
}

\begin{abstract}
We propose to use a quantum ratchet to transport quantum information in a chain of atoms trapped in an optical superlattice. The quantum ratchet is created by a continuous modulation of the optical superlattice which is periodic in time and in space. Though there is zero average force acting on the atoms, we show that indeed the ratchet effect permits atoms on even and odd sites to move along opposite directions. By loading the optical lattice with two-level bosonic atoms, this scheme permits to perfectly transport a qubit or entangled state imprinted in one or more atoms to any desired position in the lattice. From the quantum computation point of view, the transport is achieved by a smooth concatenation of perfect swap gates. We analyze setups with noninteracting and interacting particles and in the latter case we use the tools of optimal control to design optimal modulations. We also discuss the feasibility of this method in current experiments.
\end{abstract}

\section{INTRODUCTION}

By a ratchet effect one usually refers to the existence of directed transport in a system in which there is no net bias force. Ratchets have been traditionally found in the study of dissipative systems [1], where external fluctuations causing Brownian motion cooperate with a periodic force to bias transport, a mechanism that is in the basis of some biological motors [2]. Ratchets can also appear without dissipation. These Hamiltonian or conservative ratchets are interesting as they can be extended to quantum mechanical systems. In this context one finds studies that relate the existence of transport to classical properties of the model, such as some asymmetries of the external force [3] or mixing of chaotic and regular phase space regions [4].

Ultracold atoms offer an ideal arena to test this phenomenology, as can be seen from the experiments implementating both dissipative and conservative ratchets [5, 6, 7, 8, 9, 10, 11, 12, 13, 14, 15]. These experiments rely on the force imparted by near or far from resonance laser beams which act on the atoms over short periods of time. These flashing potentials implement variants of the $\delta$-kicked rotor model and lead to phenomena such as dynamical localization. On the theoretical side we must remark the discovery of directed transport on quantum mechanical systems whose classical counterpart is completely chaotic [16, 17, 18, 19, 20].

The present work aims at exploiting the fact that quantum ratchets can be used to transport quantum states and thus distribute entanglement between distant nodes, a basic task for quantum information and computation purposes 21]. Within this context, we find two distinct research directions. On the one hand, entanglement swapping and quantum teleportation 22 can be used to build efficient quantum repeaters [23, 24] for long distance communication. On the other hand, on a smaller
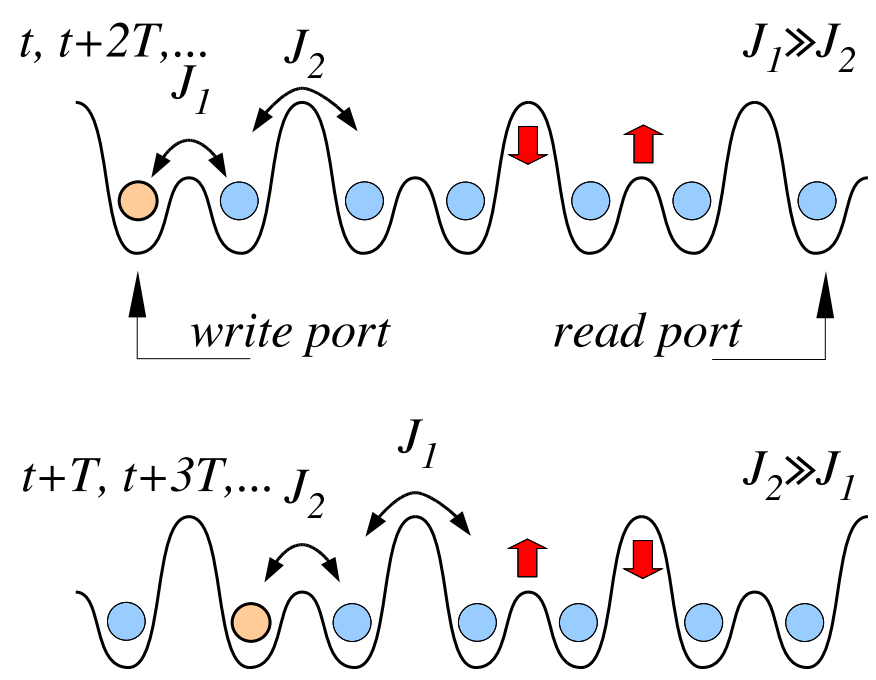

FIG. 1: (Color online)An optical superlattice arises from a combination of two potentials with different periods. By modulating the depths and displacements of these potentials we can raise and lower the tunneling rates between odd and even pairs of sites (red arrows). This way, by means of perfect swaps, the state of a particle can be transported along the lattice.

scale, a relevant effort has been recently devoted to study quantum state transfer using static local Hamiltonians which act on chains of spins, harmonic oscillators, or bosons in optical lattices 25, 26, 27, 28, 29, 30, 31, 32, 33, 34, 35, 36, 37, 38, 39, 40]. Compared to these works, time-dependent (non-adiabatic) models, such as the one presented here, provide more degrees of freedom to optimize the efficiency, speed and robustness of the quantum transport.

Moreover, the scheme we propose here for quantum state transport can be seen as a quantum ratchet induced without breaking translational and time reversal 
symmetries in the forces. The transport is just triggered by the symmetry breaking of the initial state. We illustrate this with a particular and simple implementation of quantum state transport based on two-level bosonic ultracold atoms in optical superlattices. More specifically the transport is achieved as follows. All the atoms are initially prepared in the, say, down state. At a given site (write port) an arbitrary qubit state is imprinted in the atom. The trapping potential is then modulated smoothly and periodically in time and in space. Depending on the initial asymmetric position of the atom, being in an even or odd lattice site, the qubit state is transported rightwards or leftwards to any desired lattice site (read port) [Fig. 1] without precise individual addressing [53]. We consider both free and interacting particles. To our knowledge, this is the first time many-body interactions are taken into account in Hamiltonian ratchets.

From the implementation point of view, this work is inspired by recent advances in the control and manipulation of optical superlattices [41, 42, 43, 44, 45]. Present experiments can create periodic potentials such as the ones depicted in Fig. 1, achieving a great control on the time-dependence of the potential heights, and even being able to measure the number of atoms on the even and odd sites [41, 42]. These tools suffice for the protocol devised in this work.

The outline of the paper is as follows. In Sect. III we introduce our system, made of a chain of atoms in an optical superlattice. We state the goal of this work, which is to transfer a quantum state between two arbitrary lattice sites using a translationally invariant modulation of the superlattice, and we present the Bose-Hubbard Hamiltonian that models the dynamics of the atoms. In Sect. III we study the case of noninteracting particles and find that it is possible to induce directed transport with arbitrary speed. In Sect. IV] we discuss a more realistic setup in which atoms interact. We design a ratchet Hamiltonian with the help of optimal quantum control, and find that we can still induce perfect quantum transport with a speed limited by the interaction strength. The details of the optimal quantum control technique are left to Sect. VI. In Sect. $\nabla$ we study the optical superlattice modulation in more detail, discussing the experimental challenges for implementing these quantum ratchets in current experiments. Finally, in Sect. VII we summarize our results and comment on possible extensions.

\section{OPTICAL SUPERLATTICES}

\section{A. The model}

In this work we consider the setting of ultracold neutral atoms trapped in an optical superlattice. An optical lattice is a standing wave of coherent off-resonance light created by the interference of two or more laser beams. As explained in Ref. [46], such standing wave behaves as a periodic potential that confines the atom in the minima or maxima of intensity. The optical superlattice arises from a combination of two potentials with different periods. In the simplest case the superlattice potential can be written as follows

$$
\begin{aligned}
V(x, y, z, t) & =V_{x}(t) \cos ^{2}(k x)+V_{2}(t) \cos ^{2}(2 k x+\phi)+ \\
& +V_{\perp}\left[\cos ^{2}(k y)+\cos ^{2}(k z)\right] .
\end{aligned}
$$

Here $V_{x}(t)$ and $V_{\perp}$ are the strengths of the lattice along the main axis and transversely to it, and $k=2 \pi / \lambda$ is the momentum of the photons. In order to have a configuration of decoupled 1D lattices [47], the transverse potential $V_{\perp}$ has to be much larger than the recoil energy $E_{r}=\hbar^{2} k^{2} / 2 m$ to prevent tunneling between 1D lattices. On top of this potential and along the main axis we find another lattice of strength $V_{2}(t)$, with half the period of the original one and a possible dephasing $\phi \in \mathbb{Z} \times \pi / 2$. This yields to the optical superlattice that creates the double-well structure shown in Fig. 1.

We are interested in the regime of deep lattices in which atoms are confined to the lowest Bloch band of the periodic potential. We assume the atoms to be bosons with two internal states. Under those circumstances the dynamics of the atoms can be modeled using a BoseHubbard Hamiltonian [46] which, for a single 1D tube of lattices, has the form

$$
H=\sum_{\sigma=\uparrow, \downarrow} \sum_{i=1}^{L}\left[-J_{i}\left(a_{\sigma i}^{\dagger} a_{\sigma i+1}+\text { H.c. }\right)+\frac{U}{2} a_{\sigma i}^{\dagger 2} a_{\sigma i}^{2}\right] .
$$

Here, $a_{\sigma i}$ and $a_{\sigma i}^{\dagger}$ are Fock operators that annihilate and create particles on the $i$-th site of the lattice and in one of two internal states, $\sigma \in\{\uparrow, \downarrow\}$. The parameter $U$ is the on-site interaction between atoms, which we assume to be spin independent, and $J_{i}$ is the tunneling amplitude, which may vary from site to site.

In order to describe the dynamics of the atoms, Eq. (2) has to satisfy two conditions. First of all, the tunneling $J_{i}$ between different wells has to be small, so that there effectively exists a low energy band formed by the localized states on each site. This implies that the superlattice cannot drop the barrier between pairs of sites too much [Fig. 1, for otherwise we would have to use a multiband model. The second condition is that the on-site interaction $U$ must be small compared to the energy gap between Bloch bands. Both condition are easily met in current experiments with optical superlattices [41, 43, 44, 45], and have been taken into account throughout this work [See Sect. V].

\section{B. The goal: quantum communication}

Our objective is as follows. We initially prepare all the two-level atoms in the same state, say the down state $|\downarrow\rangle$. We imprint an arbitrary quantum state on a particular site of the lattice, the write port, in such a way that the atom ends up in a superposition of both spin states, 
$\alpha|\uparrow\rangle+\beta|\downarrow\rangle$. The goal is to create, by modulating the energy barriers of the optical superlattice, a ratchet effect that will transport this state a predetermined distance, to another site, the read port in Fig. 1. The modulation of the superlattice will not break the translational invariance and it will also be periodic in time (with a period equal to $2 T$ ), alternating the roles of the hopping between even $\left(J_{2 n}=J_{2}\right)$ and odd $\left(J_{2 n+1}=J_{1}\right)$ sites

$$
J_{1,2}(t+T)=J_{2,1}(t) .
$$

The Hamiltonian in Eq. (2), being translationaly invariant in time and in space, does not exert any force on the atoms, as expected from a quantum ratchet. This can be verified by computing the integral of the forces acting on an atom in a well of the lattice. In particular, the following average is zero

$$
\int_{0}^{2 T} d t \int_{0}^{\frac{\pi}{2 k}} d x \frac{d}{d x} V(x, y, z, t)=0 .
$$

Note however that our superlattice does not have a permanent asymmetry on the unit cell $[0, \pi / 2 k)$, unlike other Hamiltonian flashed ratchets with saw-tooth profiles [8].

While the average force acting on the atoms is zero, we will show that there is still transport. The reason is the asymmetry of the initial state

$$
|\psi(0)\rangle=\left(\alpha a_{\uparrow \text { in }}^{\dagger}+\beta a_{\downarrow \text { in }}^{\dagger}\right) \prod_{j \neq \text { in }} a_{\downarrow j}^{\dagger}|\mathrm{vac}\rangle,
$$

and the fact that our time-dependent Hamiltonian will perform perfect swaps between neighboring lattice sites. All of this leads, after $n$ swaps of duration $T$, to a propagation of the imprinted qubit rightwards or leftwards

$$
|\psi(n T)\rangle=\left(\alpha a_{\uparrow \text { out }}^{\dagger}+\beta a_{\downarrow \text { out }}^{\dagger}\right) \prod_{j \neq \text { out }} a_{\downarrow j}^{\dagger}|\mathrm{vac}\rangle,
$$

depending on the starting site. Note also that by design, we will achieve the transport of the quantum state without actually moving any atom, thus leaving the number of atoms per site invariant.

Finally, let us mention that this scheme can also be used to distribute entanglement in the same fashion as in [27]. Namely by imprinting a maximally entangled state in two neighboring lattice sites. Since atoms in odd and even sites move on opposite directions, the atoms will depart from each other and entanglement will get distributed between arbitrarily distant lattice sites.

\section{NONINTERACTING CASE}

We start with the case of noninteracting particles $U=0$. This case is relatively easy to study as all particles can be treated independently and therefore we can focus on the dynamics of a single particle that starts from different sites, a problem which is integrable. The basis of states is now denoted by $|j\rangle:=a_{j}^{\dagger} \mid$ vac $\rangle$, where we no longer care about the internal state of the particle. Our only concern is now to ensure that the state with a particle on the write port $\mid j=$ in $\rangle$ is mapped after a given time, $t_{r}$, to a state with that particle on site $\mid j=$ out $\rangle$, the reading port, in a deterministic fashion. Note also that since particles do not interact we do not have to consider the dynamics of particles on other lattice sites. Nevertheless, as we show below, it is possible to look for solutions that do not give energy to these other particles and leave the density profile invariant. If the number of atoms per lattice site is constant, we can then talk about transport of the quantum state and not of particles themselves [Fig. 1].

As described before, we will adopt two restrictions. The first one is that the couplings do not break the translational invariance but are modulated in time, with the roles of $J_{2}$ and $J_{1}$ being exchanged after a time $T$, as from Eq. (3). The second one is to assume that the tunneling can be brought to zero, so that

$$
\begin{aligned}
J_{1}(t) \geq 0, J_{2}(t) & =0, \quad t \in[0, T), \\
J_{1,2}(t+T) & :=J_{2,1}(t) .
\end{aligned}
$$

During the time in which $J_{2}=0$ we have $(\hbar=1)$

$$
\left(\begin{array}{c}
a_{2 n+1}(t) \\
a_{2 n+2}(t)
\end{array}\right)=\left\{\cos [\theta(t)] \mathbb{I}+i \sin [\theta(t)] \sigma^{x}\right\}\left(\begin{array}{c}
a_{2 n+1}(0) \\
a_{2 n+2}(0)
\end{array}\right)
$$

where $\sigma^{x}$ is a Pauli matrix and $\theta(t)=\int_{0}^{t} J_{1}(\tau) d \tau$. The simplest solution of this kind is a square signal, $J_{1}(t)=J$, as shown in Fig. 2 2 b. This means that for $T=\pi /(2 J)$ we achieve a perfect swap between even and odd sites up to an irrelevant global phase. In practice the tunnelings $J_{1}$ and $J_{2}$ will evolve smoothly and require some time to reach a nonzero value. In that case the time for perfect switch will be given by $\theta(T)=\pi / 2$.

It is possible to construct arbitrarily fast solutions of also arbitrary smoothness. Note, however, that the value of the average hopping is inversely proportional to the modulation period, $\bar{J} \propto 1 / T$, so that a fast solution may require a lattice with an unrealistically large hopping.

\section{INTERACTING CASE}

The case of interacting particles is more realistic but also more difficult. We can no longer regard the particles as independent and collisions can affect the phase of the transported state, as defined by Eq. (5). We will still look for simple solutions that concatenate a swap dynamics on pairs of lattice sites, with the restrictions introduced in Sec. IIB.

\section{A. A two-level problem}

Let us repeat the calculation of Sect. III but now taking into account the interaction between particles. Since 
a)
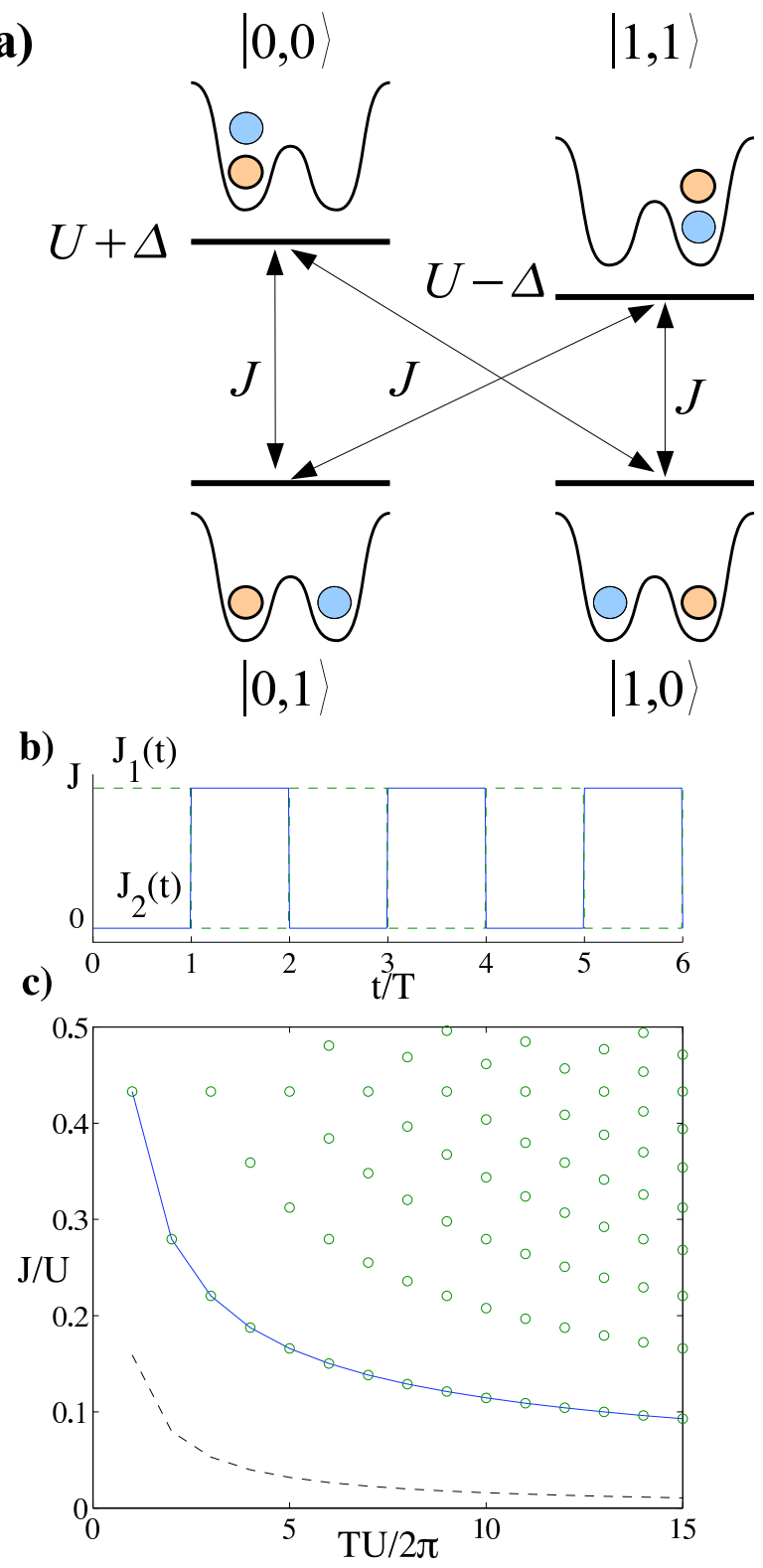

FIG. 2: (Color online) (a) Model case of two lattice sites disconnected from the rest because $J_{2}=0$. By fixing the hopping between wells to a precise value $J$ for a time $T$, it is possible to swap the atoms. Note that the doubly occupied sites have more energy due to the interaction, $U$, and a possible inhomogeneity of the lattice potential, $\Delta$. (b) Combining the solution for a pair of sites we obtain one possible modulation of the hoppings $J_{1}$ (solid) and $J_{2}$ (dashed), namely $J_{1}$ and $J_{2}$ are square waves in antiphase. This produces the quantum transport. (c) Values of $J$ and $T$ for which perfect transport is achieved. Each circle is a solution; the solid line joins the solutions with smallest hopping and the dashed line is the solution for noninteracting particles.

$J_{2}=0$ we focus on the double-well problem with only two distinguishable particles, i.e. in two different internal states. Instead of using second quantization we denote their state as $|i\rangle|j\rangle$, where $i, j \in\{0,1\}$ is the well in which the particle resides. The connections between states are depicted in Fig. 2a. Using Pauli operators the effective Hamiltonian becomes [54]

$$
H=-J\left(\sigma^{x} \otimes \mathbb{I}+\mathbb{I} \otimes \sigma^{x}\right)+\frac{U}{2}\left(\sigma^{z} \otimes \sigma^{z}+1\right)
$$

We can further restrict our problem to the only states that participate on the dynamics

$$
\begin{aligned}
\left|\psi^{-}\right\rangle & :=\frac{1}{\sqrt{2}}(|01\rangle-|10\rangle), \\
\left|\psi^{+}\right\rangle & :=\frac{1}{\sqrt{2}}(|01\rangle+|10\rangle), \\
\left|\phi^{+}\right\rangle & :=\frac{1}{\sqrt{2}}(|00\rangle+|11\rangle) .
\end{aligned}
$$

Using this basis we obtain the effective Hamiltonian

$$
H(t)=\left(\begin{array}{ccc}
0 & 0 & 0 \\
0 & 0 & -2 J(t) \\
0 & -2 J(t) & U
\end{array}\right),
$$

where $\left|\psi^{-}\right\rangle$is shown to be a dark state and the other two are coupled by a two-level Hamiltonian $2 J(t) \sigma^{x}+U \sigma^{z}$. Our problem is thus to find a hopping $J(t)$ such that after a time $T$ the states above have experienced the following transformation

$$
\left|\psi^{-}\right\rangle \rightarrow\left|\psi^{-}\right\rangle,\left|\psi^{+}\right\rangle \rightarrow-\left|\psi^{+}\right\rangle,\left|\phi^{+}\right\rangle \rightarrow e^{i \nu}\left|\phi^{+}\right\rangle,
$$

where the phase $\nu$ is unimportant for our purposes.

\section{B. The square signal revisited}

We will first investigate solutions which are piecewise constant, with $J_{1}(t)=J$, for $t \in[0, T)$. For a fixed hopping our Hamiltonian has two nonzero eigenvalues

$$
E_{ \pm}=\frac{U \pm \sqrt{16 J^{2}+U^{2}}}{2} .
$$

that contribute to the evolution of the symmetric states. The perfect swap between the atoms takes place at a time $T$ such that the symmetric state $\left|\psi^{+}\right\rangle$changes sign. As $\left|\psi^{+}\right\rangle$can be written as a superposition of the two symmetric eigenstates with energies $E_{ \pm}$, this condition is satisfied when $E_{ \pm} T= \pm\left(2 n_{ \pm}+1\right) \pi$, where $n_{+}$and $n_{-}$ are arbitrary integers. Defining $x:=\left(2 n_{+}+1\right) /\left(2 n_{-}+1\right)$ we obtain

$$
\frac{U}{J}=2 \frac{x-1}{\sqrt{x}} .
$$

From this value we can compute the energies $E_{ \pm}$and the time $T$. As shown in Fig. 2rc the minimal value of $J$ is reached for either $n_{+}$or $n_{-}$equal to 0 and, unlike in the noninteracting case, there exists a minimal swap time given by $T=2 \pi / U$. Indeed, using the tools in [48] one can prove that the constant hopping is the fastest solution and that the gate cannot be performed faster than this time. 


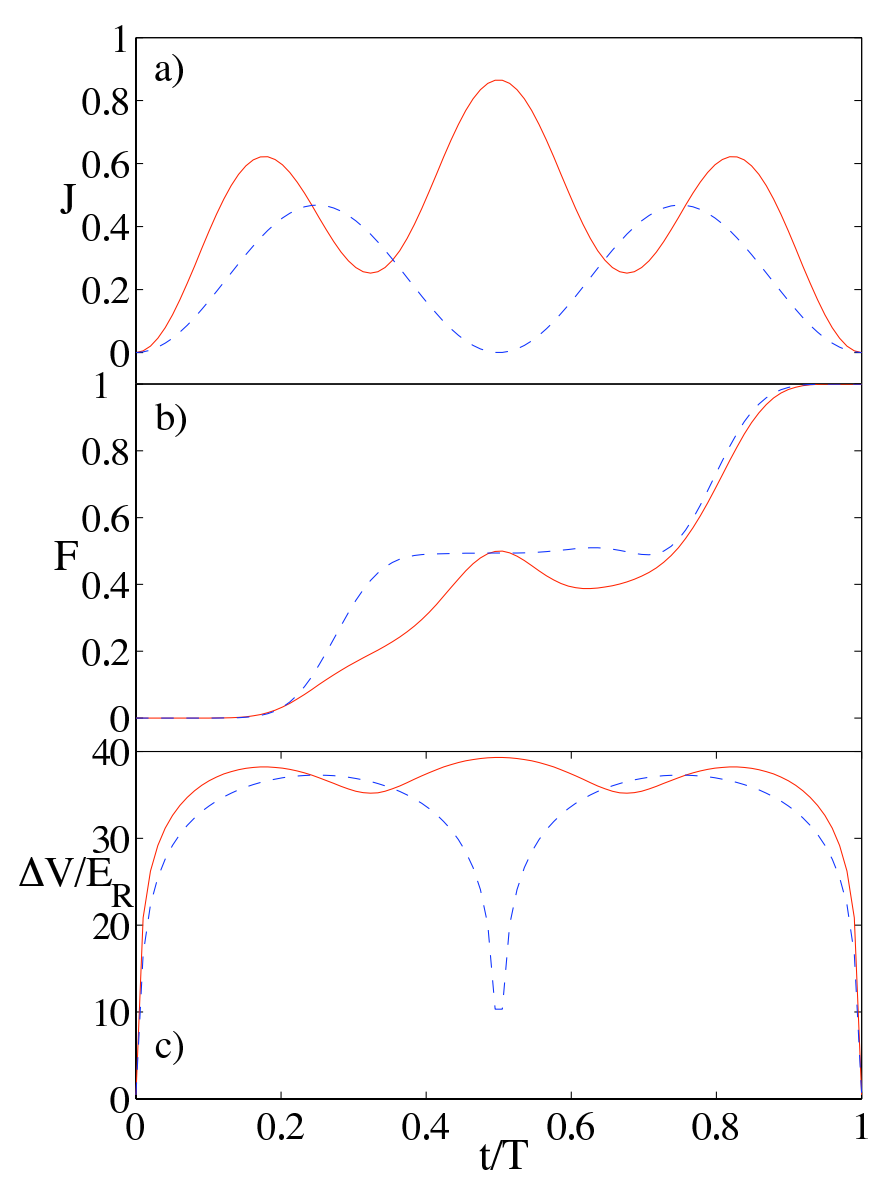

FIG. 3: (Color online)(a) Hopping and (b) fidelity of the gate $\left(F=|\langle 01|U(t)| 10\rangle|^{2}\right)$ with the perfect transport for half a period $[0, T)$ during which $J_{1}(t)=J(t)$ and $J_{2}=0$. We find two solutions, one for $T=2 \pi / U$ (solid) and another one for $T=4 \pi / U$ (dashed), with three and two modes, respectively. In (c) we plot the corresponding modulations of the superlattice.

\section{Smooth solutions: optimal quantum control}

While optimal, the square signal that we have considered before is probably unrealistic, as in experiments the hopping will smoothly increase and decrease as the tunneling barriers are changed. For that reason we have investigated other solutions with continuous derivatives using the tools of optimal quantum control.

The details of the method are left for Sect. VI, but let us sketch the procedure. The first step is to parametrize the hopping as a linear combination of some functions

$$
J(t)=\sum_{n} c_{n} f_{n}(t) \geq 0
$$

where for simplicity we use trigonometric functions $f_{n}(t)=\sin ^{2}(\pi n t / T)$ that increase and decrease smoothly to zero. Since the hopping is positive we have a first restriction, $c_{n} \geq 0$. The second restriction is that the fidelity of the swap procedure has to be one. Both constraints are imposed to the problem of minimizing the

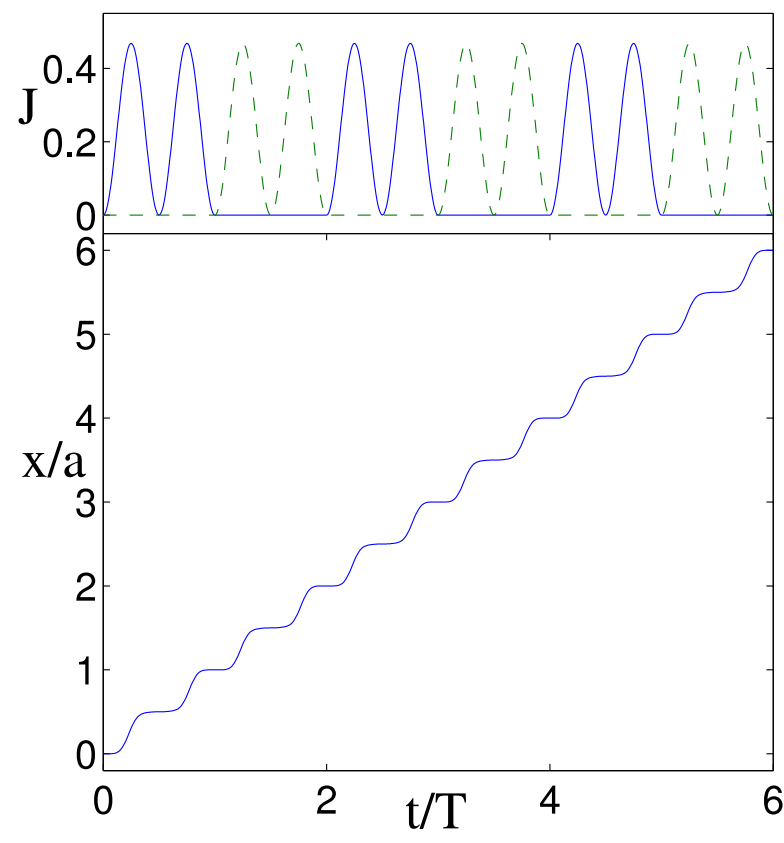

FIG. 4: (Color online)On the upper figure we plot a solution of $J_{1}(t)$ (solid) and $J_{2}(t)$ (dashed) for a perfect transport of the qubit. In the lower figure we plot the average position of the qubit on the lattice as transported by these modulations. These plots have been computed using $T=4 \pi / U$.

average strength of the hopping, given by $E=\sum_{n}\left|c_{n}\right|^{2}$. This problem is solved numerically with MATLAB's optimization toolbox [49] using as aid the derivatives computed by means of perturbation theory [Sect. VI].

In Fig. 3 we show two instances of the problem, one optimized for three modes $\left(c_{n}=0\right.$ for $\left.n>3\right)$ and a duration of $T=2 \pi / U$, the other one for twice the time and two modes. As it can be appreciated in the picture, we reach perfect fidelity in a rather smooth and robust manner, so that errors in the timing of the gate will not affect the process significantly.

To further relate these solutions to the notion of a ratchet let us look at Fig. 4. There we plot the full time evolution of the imprinted qubit state for 6 periods of an optimal modulation. The position may oscillate between pairs wells, but there is always a net average transport.

\section{Dealing with holes}

The two solutions derived above, that is the piecewise constant and the smooth ones, are designed to induce transport on a chain of particles. However, in practice such a chain will have some endpoints or particles that stand near an empty site. We can then have three scenarios: (i) that a particle standing near a hole ends up in the original site at time $t=T$; (ii) that the particle and the hole are swapped and, more generally, (iii) that the particle and the hole end up in some coherent superposition of being on each site. Out of these processes, only 


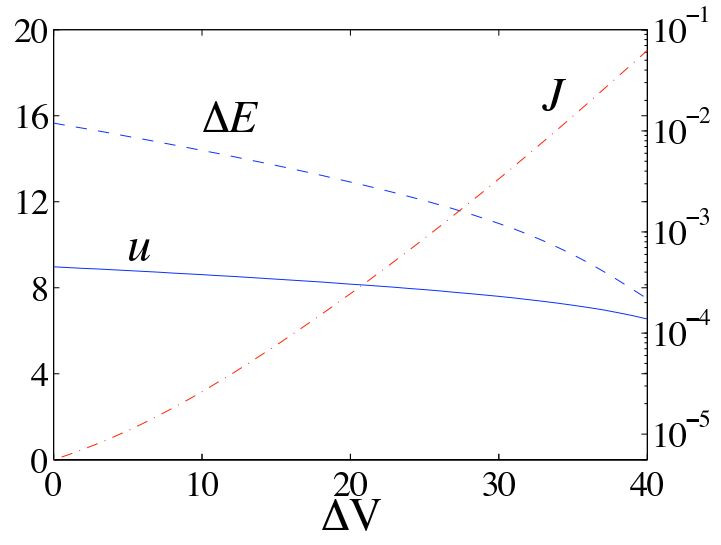

FIG. 5: (Color online)Parameters of the double well potential as a function of the superlattice modulation, $\Delta V=\left(V_{x}+\right.$ $\left.V_{2}\right) / 2$. We plot the effective hopping between wells, $J$ (dashdot, right axis), the energy gap to higher bands, $\Delta E$ (dashed), and the on-site interaction, $u$ (solid). Everything is expressed in units of the recoil energy, $E_{r}$.

the latter will affect the evolution of the state we want to transport, since there is a small probability that it gets reflected.

Holes only have a disturbing effect on the transport if $U \neq 0$, since in the noninteracting case surrounding particles are equivalent to holes. Note however that we can effectively eliminate the scenario (iii) if we impose that the hopping on half a period leaves the particle invariant

$$
\int_{0}^{T} J(\tau) d \tau=2 \pi \times \mathbb{Z}
$$

While this restriction cannot always be achieved for the piecewise constant profile, it can be easily incorporated to our optimal control toolbox.

\section{EXPERIMENTAL IMPLEMENTATION}

All the protocols that we have designed can be implemented in current experiments with optical superlattices [41, 42, 43, 44, 45]. The implementation should begin by loading the superlattice with approximately one atom per site, all of them in the same internal state. The next step is to rotate the state of some atoms using either magnetic field gradients, coherent light or a clever combination of both [50], in order to imprint the quantum state we wish to transport. By modulating in time the intensity of the laser beams that participate in the optical lattice one can then achieve a modulation of $J_{1}(t)$ and $J_{2}(t)$ that corresponds to the solutions studied above. After an appropiate time one may retrieve the qubit state by measuring the lattice sites on which it is expected to arrive.

In an experiment one does not directly control the hoppings $J_{1}(t)$ and $J_{2}(t)$, but rather the lattice strengths $V_{x}$ and $V_{2}$ [See Eq. (1)]. In order to relate these two quantities we have have performed a band structure calculation. We have focused on the case $J_{2}(t) \simeq 0$, which corresponds to the first half-period, $t \in[0, T)$. This hopping can be suppressed by making $\phi=0$ and $V_{x}+V_{2}=70 E_{r}$, which is large enough to effectively suppress hopping every second site. Given this constraint, any modulation of the lattices depends on a single quantitiy, $\Delta V(t) \geq 0$, such that $V_{x}=\Delta V(t)$ and $V_{2}=70 E_{r}-\Delta V(t)$. It thus remains to relate $\Delta V(t)$ and $J_{1}(t)$. This is done for each possible value of $\Delta V$, by computing numerically the ground state wavefunction of a particle in a double-well, $w(x)$, and the first excited state. The energy difference between these states is proportional to the effective tunneling $J_{1}(t)$, while the on-site interaction energy becomes

$$
\frac{U}{E_{r}}=\frac{a_{s}}{a} u
$$

where $a_{s}$ is the scattering length between atoms, $a$ the period of the superlattice and $u=\int|w(x)|^{4}$.

The results are shown in Fig. 5. Since $a_{s} / a$ is very small, the on-site interaction energy is smaller than the energy gap between Bloch bands and the Bose-Hubbard model is therefore valid throughout the evolution (2). Furthermore, $u$ does not change very much as a function of the modulation $\Delta V$, while $J$ decays exponentially fast with $\Delta V$. After an appropiate fit of these quantities, one may convert the time dependence of $J_{1}(t)$ from Fig. $3 \mathrm{a}$ into the associated modulations of the superlattice $\Delta V(t)$, which are shown in Fig. 3 t.

\section{COHERENT CONTROL}

In this section we present the tools that we have used to optimize the modulation of the hopping, as they differ significantly from what is the standard approach in optimal quantum control based on a Lagrangian formulation 51].

\section{A. Objective function}

Let us formulate our problem: we have a Hamiltonian, $H(t ; x)$ that depends both on time and on some additional parameters, $x_{1} \ldots x_{M}$. Our goal is to find an optimal set of parameters of the Hamiltonian such that the evolution of a number of states is as equivalent as possible to a transformation given by a specific unitary $U_{g}$.

Stated in a more concrete way, the evolution of an arbitrary state $|\psi(0)\rangle$ is given by $|\psi(t)\rangle=U(t ; x)|\psi(0)\rangle$, where the unitary is a solution of the Schrödinger equation

$$
i \frac{d}{d t} U(t ; x)=H(t ; x) U(t ; x)
$$


with initial condition $U(0 ; x)=\mathbb{I}[55]$. Our goal is to maximize the fidelity (defined below) of any evolved state $U(t ; x)\left|\psi_{n}\right\rangle$ with the desired transformed state $U_{g}\left|\psi_{n}\right\rangle$. There are many ways to measure the accuracy of the transformation. A strict and simple objective function is the fidelity

$F=\frac{1}{d} \operatorname{Re}\left\{\operatorname{tr}\left[U_{g}^{\dagger} U(T ; x)\right]\right\}=\frac{1}{d} \operatorname{Re} \sum_{n=1}^{d}\left\langle\psi_{n}\left|U_{g}^{\dagger} U(T ; x)\right| \psi_{n}\right\rangle$,

where the $\left\{\left|\psi_{n}\right\rangle\right\}$ form an orthonormal basis of the $d$ dimensional Hilbert space $\mathcal{H}$ where we want to control the evolution [56]. This function is bounded by $F \in$ $[-1,1]$ and achieves the maximum value for the perfect transformation,

$$
F=1 \Leftrightarrow U(T ; x)=U_{g}
$$

The question is thus, how do we maximize $F$ ? A natural way is to compute (when possible) the derivative of $F$ with respect to the parameters $x$, i.e. $\partial F / \partial x_{i}$, since the gradient itself provides a direction along which the fidelity is increased. Indeed, given this derivative there are multiple optimization algorithms that would allow us to compute the optimal control.

\section{B. Formal gradient}

In order to obtain the gradient of $F$ with respect to the parameters $x$, we straightforwardly obtain from (19)

$$
\frac{\partial F}{\partial x_{i}}=\frac{1}{d} \operatorname{Re} \sum_{n}\left\langle\psi_{n}\left|U_{g}^{\dagger} \frac{\partial}{\partial x_{i}} U(T ; x)\right| \psi_{n}\right\rangle,
$$

which relates the gradient of $F$ to a derivative of the unitary operator. What follows is a simple way to compute $\partial U / \partial x_{i}$ which is based on performing a Taylor expansion of the operator $U(t ; x)$ with respect to the parameters $x$

$$
U(t ; x+\epsilon)=U(t ; x)+\epsilon \frac{\partial U}{\partial x}(t ; x)+\mathcal{O}\left(\epsilon^{2}\right) .
$$

We will obtain this series using time-dependent perturbation theory on the Schrödinger equation (18), which will enable us to identify the derivative of the unitary operator.

Let us assume that by changing $x \rightarrow x+\epsilon$ our Hamiltonian decomposes into an unperturbed part, $H_{0}=H(t ; x)$ and a perturbation $H_{1}$

$$
\epsilon H_{1} \equiv H(t ; x+\epsilon)-H_{0}=\epsilon \frac{\partial H}{\partial x}(t ; x)+\mathcal{O}\left(\epsilon^{2}\right) .
$$

The new unitary operator will satisfy a Schroödinger equation with a modified Hamiltonian

$$
i \frac{d}{d t} U(t ; x+\epsilon)=\left(H_{0}+\epsilon H_{1}\right) U(t ; x+\epsilon),
$$

and same initial condition $U(0 ; x+\epsilon)=\mathbb{I}$. It is now convenient to move to the interaction picture

$$
U(t ; x+\epsilon) \equiv U(t ; x) W(t ; x),
$$

which leads to a simpler equation

$$
i \frac{d}{d t} W(t ; x)=\epsilon U(t ; x)^{\dagger} H_{1}(t ; x) U(t ; x) W(t ; x) .
$$

Integrating formally this differential equation and iterating the resulting formula, one obtains the usual Dyson series. We only need this series up to first order

$$
W(t ; x)=\mathbb{I}-i \epsilon \int_{0}^{t} d \tau U(\tau ; x)^{\dagger} H_{1}(t ; x) U(\tau ; x)+\mathcal{O}\left(\epsilon^{2}\right) .
$$

From (25) combined with (27) we have

$$
\begin{aligned}
U(t ; x+\epsilon) & =U(t ; x)- \\
& -i \epsilon U(t) \int_{0}^{t} d \tau U(\tau)^{\dagger} H_{1}(\tau) U(\tau)+\mathcal{O}\left(\epsilon^{2}\right)
\end{aligned}
$$

(In the second term and hereafter we omit the $x$ dependence to ease the notation). We can now compare this expression with (22) in order to identify the derivative of the unitary, that is

$$
\frac{\partial}{\partial x} U(t)=-i \epsilon U(t) \int_{0}^{t} d \tau U(\tau)^{\dagger} \frac{\partial H}{\partial x}(\tau) U(\tau) .
$$

Therefore we obtain the formula for the gradient of the fidelity

$$
\frac{\partial F}{\partial x_{i}}=\frac{1}{d} \operatorname{Re} \sum_{n} \int_{0}^{T} d \tau\left\langle U_{g}^{\dagger} U(T) U(\tau)^{\dagger} \frac{\partial H}{\partial x_{i}}(\tau) U(\tau)\right\rangle_{\psi_{n}} .
$$

\section{Development of the algorithm}

Even though we have a closed expression for the derivative of the fidelity, we still need to compute the integral which appears in Eq. (30). We have devised a simple, accurate and efficient procedure which is based on solving three sets of ordinary differential equations. The first one is obtained by transforming the integral in Eq. (30) into $d \times M$ ordinary differential equation

$$
\frac{d}{d t} f_{n, i}(t)=\frac{1}{d} \operatorname{Re}\left\langle\psi_{n}\left|U_{g}^{\dagger} U(T) U(t)^{\dagger} \frac{\partial H}{\partial x_{i}}(t) U(t)\right| \psi_{n}\right\rangle
$$

with initial condition $f_{n, i}(0)=0$ and final value

$$
\frac{\partial F}{\partial x_{i}}=\sum_{n} f_{n, i}(T) .
$$

We now notice that left side of the scalar product in Eq. (31) is the state $U(t) U(T)^{\dagger} U_{g}\left|\psi_{n}\right\rangle$ and can be computed by solving an ordinary differential equation

$$
i \frac{d}{d t}\left|\xi_{n}(t)\right\rangle=H(t)\left|\xi_{n}(t)\right\rangle,
$$


with initial condition $\left|\xi_{n}(T)\right\rangle=U_{g}\left|\psi_{n}\right\rangle$ and moving backwards in time from $T$ to $t$.

We now have all the ingredients to design the protocol that computes our derivative (30). We summarize it as follows. First, solve Eq. (33) backwards in time up to $t=0$, finding

$$
\left|\xi_{n}(0)\right\rangle:=U(T)^{\dagger} U_{g}\left|\psi_{n}\right\rangle .
$$

We then solve the system of ordinary differential equations

$$
\begin{aligned}
i \frac{d}{d t}\left|\psi_{n}(t)\right\rangle & :=H(t)\left|\psi_{n}(t)\right\rangle \\
i \frac{d}{d t}\left|\xi_{n}(t)\right\rangle & :=H(t)\left|\xi_{n}(t)\right\rangle \\
\frac{d}{d t} f_{n, i} & :=\frac{1}{d} \operatorname{Im}\left\langle\xi_{n}(t)\left|\frac{\partial H}{\partial x_{i}}(t)\right| \psi_{n}(t)\right\rangle .
\end{aligned}
$$

With the initial conditions $\left|\psi_{n}(0)\right\rangle$ being some orthonormal basis of our Hilbert space, $\left|\xi_{n}(0)\right\rangle$ computed before and $f_{n, i}(0)=0$. The value of the derivative is then computed using Eq. (32).

This derivative and the formulas given above can be fed to any optimization package, such as a simple line search algorithm or a nonlinear conjugate gradient method. In particular we have used Matlab's nonlinear optimization toolbox [49].

\section{CONCLUSIONS}

In this work we have proposed to generate quantum transport using cold atoms in an optical superlattice that is modulated periodically both in time and in space. Since there is no average force acting on the atoms, we deal with a quantum ratchet effect that makes atoms on even and odd sites move along opposite directions.

We have demonstrated that the ratchet effect can be induced both for free particles and in the case of nonzero on-site interactions. The latter represents to our knowledge the first time strong many-body interactions have been treated exactly in Hamiltonian ratchets [57]

This ratchet effect can be used to transport quantum information by imprinting a qubit or an entangled state on one or more atoms of the atomic chain and letting the system evolve according to our ratchet potentials. The dynamics generated with our scheme corresponds to a smooth concatenation of perfect swap gates. Therefore, after a time $n \times T$, we will find that the qubit state has been transported a well determined distance, of order $n$, along the lattice.

Our ideas could be implemented in current experiments with optical superlattices. From the quantum information point of view, compared to other proposals based on effective spin interactions between atoms, the ratchet effect should be faster and lead to a more flexible dynamics. From the fundamental point of view, we believe that the modulated superlattices are a rich playground in which to study transport phenomena, quantum diffusion and the influence of noise and of chaos in the transport of quantum states.

We thank J. Calsamiglia for useful discussions. We are grateful to A. Sanpera, R. Munoz-Tapia, and A. Kay for a careful reading of the manuscript. O.R.I. acknowledges support from the spanish MEC grants AP20050595, FIS2005-03169, Consolider-Ingenio2010 CSD200600019 QOIT, and Catalan grant SGR-00185. J.J.G.R acknowledges financial support from the Ramon y Cajal Program and from the spanish projects FIS2006-04885 and CAM-UCM/910758.
[1] P. Reimann, Phys. Rep. 290, 149 (1997).

[2] R. D. Astumian, Science 276, 917 (1997).

[3] S. Flach, O. Yevtushenko, and Y. Zolotaryuk, Phys. Rev. Lett. 84, 2358 (2000).

[4] H. Schanz, M. F. Otto, R. Ketzmerick, and T. Dittrich, Phys. Rev. Lett. 87, 070601 (2001).

[5] P. H. Jones, M. Goonasekera, and F. Renzoni, Phys. Rev. Lett. 93, 073904 (2004).

[6] F. L. Moore, J. C. Robinson, C. F. Bharucha, B. Sundaram, and M. G. Raizen, Phys. Rev. Lett. 75, 4598 (1995).

[7] B. G. Klappauf, W. H. Oskay, D. A. Steck, and M. G. Raizen, Phys. Rev. Lett. 81, 1203 (1998).

[8] C. Mennerat-Robilliard, D. Lucas, S. Guibal, J. Tabosa, C. Jurczak, J.-Y. Courtois, and G. Grynberg, Phys. Rev. Lett. 82, 851 (1999).

[9] J. Ringot, P. Szriftgiser, J. C. Garreau, and D. Delande, Phys. Rev. Lett. 85, 2741 (2000).

[10] G. Behinaein, V. Ramareddy, P. Ahmadi, and G. S. Summy, Phys. Rev. Lett. 97, 244101 (2006).

[11] R. Gommers, S. Denisov, and F. Renzoni, Phys. Rev.
Lett. 96, 240604 (2006).

[12] L. Sanchez-Palencia, Phys. Rev. E 70, 011102 (2004).

[13] P. Sjolund, S. J. H. Petra, C. M. Dion, S. Jonsell, M. Nylen, L. Sanchez-Palencia, and A. Kastberg, Phys. Rev. Let.. 96, 190602 (2006).

[14] S. Denisov, L. Morales-Molina, S. Flach, P. Hänggi, Phys. Rev. A 75, 063424 (2007).

[15] D. Poletti, G. Benenti, G. Casati, and B. Li, Phys. Rev. A 76, 023421 (2007).

[16] T. S. Monteiro, P. A. Dando, N. A. C. Hutchings, and M. R. Isherwood, Phys. Rev. Lett. 89, 194102 (2002).

[17] T. Jonckheere, M. R. Isherwood, and T. S. Monteiro, Phys. Rev. Lett. 91, 253003 (2003).

[18] N. A. C. Hutchings, M. R. Isherwood, T. Jonckheere, and T. S. Monteiro, Phys. Rev. E 70, 036205 (2004).

[19] G. Hur, C. E. Creffield, P. H. Jones, and T. S. Monteiro, Phys. Rev. A 72, 013403 (2005).

[20] A. Kenfack, J. Gong, and A. K. Pattanayak, arXiv:0708.3026 (2007).

[21] C. H. Bennett and D. P. DiVincenzo, Nature 404, 247 (2000). 
[22] C. H. Bennett, G. Brassard, C. Crepeau, R. Jozsa, A. Peres, and W. K. Wootters, Phys. Rev. Lett. 70, 1895 (1993).

[23] H.-J. Briegel, W. Dür, J. I. Cirac, and P. Zoller, Phys. Rev. Lett. 81, 5932 (1998).

[24] L.-M. Duan, M. D. Lukin, J. I. Cirac, and P. Zoller, Nature 414, 413 (2001).

[25] S. Bose, Phys. Rev. Lett. 91, 207901 (2003).

[26] T. J. Osborne and N. Linden, Phys. Rev. A 69, 052315 (2004).

[27] L. Amico, A. Osterloh, F. Plastina, R. Fazio, and G. M. Palma, Phys. Rev. A 69, 022304 (2004).

[28] M. Christandl, N. Datta, A. Ekert, and A. J. Landahl, Phys. Rev. Lett. 92, 187902 (2004).

[29] M. B. Plenio and F. L. Semiao, New J. Phys. 7, 73 (2005).

[30] A. Wojcik, T. Luczak, P. Kurzynski, A. Grudka, T. Gdala, and M. Bednarska, Phys. Rev. A 72, 034303 (2005).

[31] H. L. Haselgrove, Phys. Rev. A 72, 062326 (2005).

[32] D. Burgarth and S. Bose, Phys. Rev. A 71, 052315 (2005).

[33] D. Burgarth, PhD Thesis, arXiv:0704.1309 (2007).

[34] O. Romero-Isart, K. Eckert, and A. Sanpera, Phys. Rev. A 75, 050303 (2007).

[35] K. Eckert, O. Romero-Isart, and A. Sanpera, New J. Physics 9, 155 (2007).

[36] A. Bayat and S. Bose, arXiv:0706.4176 (2007).

[37] A. Kay, Phys. Rev. Lett. 98, 010501 (2007).

[38] I. D'Amico, B. W. Lovett, and T. P. Spiller, Phys. Rev. A 76, 030302(R) (2007).

[39] O. Romero-Isart, K. Eckert, C. Rodó, and A. Sanpera, J. Phys. A: Math. Theor. 40, 8019 (2007).

[40] S. Bose, cond-mat/0610024 (2006).

[41] S. Fölling, S. Trotzky, P. Cheinet, M. Feld, R. Saers, A. Widera, T. Müller, and I. Bloch, Nature 448, 1029 (2007).

[42] M. Anderlini, P. J. Lee, B. L. Brown, J. Sebby-Strabley, W. D. Phillips, and J. V. Porto, Nature 448, 452 (2007).
[43] P. J. Lee, M. Anderlini, B. L. Brown, J. Sebby-Strabley, W. D. Phillips, and J. V. Porto, Phys. Rev. Lett. 99, 020402 (2007).

[44] M. Anderlini, J. Sebby-Strabley, J. Kruse, J. V. Porto, and W. D. Phillips, J. Phys. B: At. Mol. Op. 39, 199 (2006).

[45] J. Sebby-Strabley, M. Anderlini, P. S. Jessen, and J. V. Porto, Phys. Rev. A 73, 033605 (2006).

[46] D. Jaksch, C. Bruder, J. I. Cirac, C. W. Gardiner, and P. Zoller, Phys. Rev. Lett. 81, 3108 (1998).

[47] M. Greiner, O. Mandel, T. Esslinger, T. W. Hänsch, and I. Bloch, Nature 415, 39 (2002).

[48] N. Khaneja, R. Brockett, and S. J. Glaser, Phys. Rev. A 63, 032308 (2001).

[49] M. A. Branch, T. F. Coleman, and Y. Li, SIAM J. Sci. Comput. 21, 1 (1999).

[50] S. Fölling, A. Widera, T. Muller, F. Gerbier, and I. Bloch, Phys. Rev. Lett. 97, 060403 (2006).

[51] J. Werschnik and E. K. U. Gross, arXiv:0707.1883v1.

[52] C. E. Creffield, Phys. Rev. Lett. 99, 110501 (2007).

[53] During the completion of this manuscript, an article of C. E. Creffield [52] with related ideas has appeared. He shows how the quantum control of a periodic driving field can be employed to guide the motion of a boson in an otherwise empty optical lattice and to create entanglement by the interaction of two distinguishable bosons.

[54] We will assume that the inhomogenity $\Delta$ in Fig. 2 is zero, because otherwise the lattice has a net bias force and we cannot talk about a "ratchet".

[55] Note that we only specify the final time, but assume that the initial time is set at $t=0$.

[56] It is also possible to perform the optimization over a smaller subspace, using fewer vectors. This is particularly useful when one wants, for instance, to control the evolution of a single state.

[57] In the work of [15] the ratchet is studied using the meanfield formalism for a Bose-Einstein condensate. 